\title{
1. Peripheral neurotomy in the neurosurgical treatment of spasticity
}

Service de Neurochirurgie, Hôspital Henri Mondor, Paris Philippe Decq

Introduced by Stoffel in 1912 for the treatment of spastic equinus foot, selective fascicular neurotomy consists of a partial section of the motor collaterals of the muscles presenting excessive spasticity. This peripheral surgery for spasticity is based on two main concepts: 1) Spinal reflexes and muscles are heterogeneous and have a regional organization depending on their contribution in normal movements. This is the physiological base of focal spasticity and peripheral treatment. 2) There is a differential re-innervation after a partial section, leading to motor recovery without spindles reinnervation (therefore without spasticity).

Before performing neurotomies, a careful clinical assessment is required: what kind of spasticity? For what kind of goal (functional or comfort)? The surgery could be prior to surgery mimicked by motor nerve blocks (anesthetic drugs)

There are a lot of neurotomies, depending of the clinical status, either in the lower or the upper limb. The most frequent is the tibial neurotomy for spastic equinus foot.

\section{Intrathecal baclofen therapy for spasticity}

Division of Neurosurgery, University of Alabama at Birmingham Mark N. Hadley

Baclofen binds to GABA receptors within the brainstem, dorsal horn of the spinal cord and other central nervous system sites. Baclofen administered via continuous infusion into the spinal subarachnoid space (intrathecal) has been proven to be effective in the treatment of spasticity and hypertonia following spinal cord injury (SCI).

Our group has administered intrathecal baclofen therapy to patients with long-standing cerebral palsy (CP) with goodlong term results and improved function. We have applied this therapy to patients with spastic-dystonia from acquired traumatic brain injury (TBI) and stroke. Ashworth scores, spasm scores, and reflex scores have been statistically significantly improved $(\mathrm{p}<0.0001)$ at one ${ }^{-}$year follow ${ }^{-}$up in these patients without diminution in existing motor function.

Continuous intrathecal infusion of baclofen is capable of maintaining a reduction in spasticity and dystonia in both the upper and lower extremities of patients with SCI, CP, TBI and stroke. Our experiences with assessment, operative techniques and follow-up will be presented. 